CAHIERS DE

NARRATOLOGIE

\section{Cahiers de Narratologie}

Analyse et théorie narratives

$38 \mid 2020$

Lusor in Fabula. Jeu vidéo et nouvelles frontières du récit

\title{
Entre règles et narration : étude narratologique des tutoriels de jeu vidéo et des " personnages-guides »
}

\section{Fanny Barnabé}

\section{(2) OpenEdition \\ Journals}

Electronic version

URL: http://journals.openedition.org/narratologie/11676

DOI: 10.4000/narratologie. 11676

ISSN: 1765-307X

Publisher

LIRCES

Electronic reference

Fanny Barnabé, "Entre règles et narration : étude narratologique des tutoriels de jeu vidéo et des « personnages-guides »", Cahiers de Narratologie [Online], 38 | 2020, Online since 18 January 2021, connection on 29 January 2021. URL: http://journals.openedition.org/narratologie/11676 ; DOl: https://doi.org/10.4000/narratologie. 11676

This text was automatically generated on 29 January 2021.

Article L.117-1 du Code de la propriété intellectuelle. 


\title{
Entre règles et narration : étude narratologique des tutoriels de jeu vidéo et des « personnages-guides »
}

\author{
Fanny Barnabé
}

\section{Introduction}

\section{Les tutoriels de jeux vidéo comme objet d'étude}

1 Inscrite au croisement des game studies, de la narratologie et de la rhétorique, la présente recherche prendra pour objet les tutoriels vidéoludiques, ces passages métadiscursifs durant lesquels les jeux transmettent aux joueurs des informations utilitaires sur leurs commandes (" appuie sur $\mathrm{X}$ pour $\mathrm{X}$ »), leurs règles («l'épée peut casser les blocs »), sur les comportements attendus du joueur (« tu peux faire X », « fais $\mathrm{X} »$ ) ou sur leurs principaux objectifs (« sauve la princesse »). Plus précisément, nous nous pencherons ici sur les incarnations narratives du tutoriel sous la forme de "personnages-guides », ces mascottes qui accompagnent la progression de l'avatar en lui dictant la marche à suivre à chaque étape du jeu. Ces êtres hybrides représentent en effet une porte d'entrée idéale pour étudier les mécanismes de narrativisation des règles qui sont à l'œuvre au sein du médium, étant donné qu'ils combinent une fonction utilitaire (révéler le mode d'emploi du jeu) et une fonction narratologique (faire progresser le récit). En d'autres mots, ces personnages exposent de manière particulièrement condensée les contraintes que le gameplay impose à la construction d'univers fictionnels vidéoludiques.

2 Cet article s'inscrit dans le cadre d'un projet de recherche plus large consacré à l'étude des tutoriels de jeu vidéo et de leurs fonctions narratives et rhétoriques. Malgré leur omniprésence dans le domaine vidéoludique, les tutoriels n'ont effectivement été qu'assez peu pris pour objet de recherche à ce jour. Les quelques travaux s'y intéressant relèvent le plus souvent des sciences du design et adoptent un point de vue prescriptif 
en tentant de déterminer ce que serait un «bon » tutoriel, un tutoriel « efficace » ou " immersif », ou encore en cherchant un moyen de se débarrasser de ce dispositif dans les jeux (voir entre autres Schell, 2008 et Koster, 2013, ou, dans le domaine spécifique de la réalité virtuelle : Raffaele, de Carvalho et Silva, 2017 ; Frommel et al., 2017 ; Morin et al., 2016 ; Andersen et al., 2012). Les sciences de l'éducation portent aussi un intérêt aux stratégies tutorielles du jeu vidéo et à leur possible adaptation en contexte d'apprentissage (pour un aperçu, voir Steinkuehler et al., 2012), tandis que d'autres textes abordent le sujet dans une perspective historique (comme chez Therrien et Julien 2015, qui retracent l'apparition des systèmes d'assistance dans les jeux et leur évolution).

3 Le caractère ponctuel de ces études a de quoi surprendre si l'on considère la centralité du tutoriel parmi les conventions vidéoludiques. En effet, contrairement aux médias non interactifs, le jeu vidéo est sommé de constamment manifester (plus ou moins explicitement) ses règles de manipulation à son récepteur, puisque l'œuvre ludique n'existe que par l'action de ce dernier (spécificité du jeu théorisée sous le concept d'« impératif d'action »; Genvo, 2008). Si peu de livres s'ouvrent sur un mode d'emploi exposant comment procéder à leur lecture, ce type d'indications constitue, au contraire, une norme dans le domaine vidéoludique. Le fait que l'apprentissage des règles s'y fasse par l'expérimentation, soit intégré à la partie (voire à la narration) et représente bien souvent la première interaction que le joueur peut avoir avec l'univers du jeu fait de ces passages un lieu nodal, où se concentrent nombre de problématiques centrales pour l'étude du médium.

4 Pour contribuer à l'exploration de ce domaine en friche, nous avons entamé la constitution d'une base de données de tutoriels de jeux vidéo, que nous exploitons en utilisant l'analyse factorielle des correspondances et qui a pour but de servir à cartographier les diverses formes que peuvent prendre ces dispositifs dans les œuvres ludiques existantes. À l'heure actuelle, cette base de données contient un total de 242 tutoriels, dont les propriétés sont étiquetées en fonction de divers critères (le tutoriel est-il limité à un niveau ou s'étend-il sur tout le jeu ? met-il la partie en pause ou non ? est-il facultatif ou obligatoire ? etc.) ${ }^{1}$. Parmi ces différents critères, deux ont trait au sujet qui nous occupe dans cet article : celui de la diégétisation (le tutoriel est-il ou non intégré à l'univers fictionnel du jeu ?) et celui du support (les informations tutorielles passent-elles par l'intermédiaire de l'interface, du décor, du paratexte ou d'un personnage ?). Si la constitution de cette base de données ne sera pas l'objet principal de la réflexion que nous développerons ici, elle a donc néanmoins permis d'isoler un sous-corpus de jeux au sein desquels le tutoriel est (au moins partiellement) intégré au monde fictionnel et est délivré par un personnage. C'est de ce corpus que seront issus les différents exemples mobilisés au cours de notre analyse.

\section{Tutoriels personnifiés, personnages-guides : des entités problématiques}

De prime abord, les tutoriels peuvent apparaitre comme des dispositifs dont la fonction est prioritairement liée au gameplay et aux règles empiriques d'utilisation du jeu, puisqu'ils servent précisément à rendre celles-ci explicites. Toutefois, dans bon nombre de jeux vidéo (et dans environ un tiers du corpus étudié), les tutoriels exercent 
également une fonction narratologique : ils sont des matrices à personnages, servant à peupler la diégèse.

Dans les jeux qui construisent un univers fictionnel, les tutoriels peuvent en effet servir d'excuse pour créer un grand nombre de "personnages-guides", c'est-à-dire de personnages dont la principale (voire la seule) fonction est de révéler des informations utilitaires au joueur. Dans le jeu d'action-RPG Zelda II: The Adventure of Link (Nintendo, 1987), par exemple, les règles d'utilisation du jeu ne sont pas prises en charge par un menu d'interface ou par un niveau d'entrainement qui précéderait le début de l'aventure, mais bien par les $\mathrm{PNJ}^{3}$ que le joueur peut croiser dans les différents villages dispersés au sein du jeu. Si ce dernier prend la peine de s'adresser à ces villageois, ceuxci répondront par une ligne de dialogue livrant des indices tels que : «Utilise les clefs dans les palais où elles se trouvent ${ }^{4}$ ", "Ne va pas au Sud sans une bougie ${ }^{5}$ ", ou encore "Quand tu sautes, appuie sur bas pour donner un coup d'épée ${ }^{6}$ ». La fonction tutorielle est donc ici ce qui confère une identité à ces figures qui, sans leurs lignes de dialogue spécifiques, se confondraient toutes (puisque, graphiquement, elles sont le fruit du recyclage d'un nombre très limité de sprites identiques).

7 En d'autres termes, l'intégration des villageois au tutoriel est ce qui fait proprement d'eux des personnages: loin d'être des images sans vie qui se confondraient avec le décor, ceux-ci sont des individus qui connaissent des informations, ce qui (malgré leur caractère basique) laisse la possibilité au joueur de leur prêter une vie propre, un passé imaginaire qui leur aurait permis d'acquérir ce savoir. À ce titre, dans Zelda II, les personnages-guides se distinguent fortement des villageois dépourvus de fonction tutorielle, qui se contentent de répéter la phrase «Désolé. Je ne sais rien ${ }^{7}$ » et qui forment donc une entité commune et indifférenciée à laquelle il est plus difficile de prêter le titre de personnage (voir Figure 1).

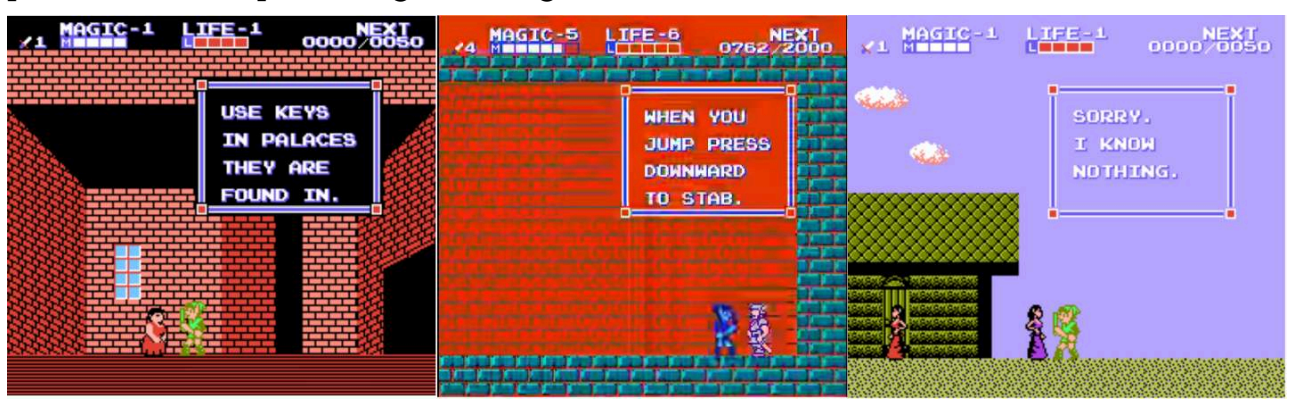

Figure (Tous Droits Réservés) - Personnages-guides dans Zelda II

8 Le tutoriel est ainsi un outil permettant de générer, à peu de frais, une multitude de voix narratives venant donner vie et sens à un univers fictionnel. Cependant, les entités créées par ce processus, ces «tutoriels personnifiés ", ne vont pas sans questionner les frontières de la notion de personnage telle qu'elle est traditionnellement entendue ainsi que les seuils des espaces diégétiques que ces figures sont censées habiter. Dans la suite de cet article, nous nous interrogerons donc sur la manière dont les discours tutoriels construisent les personnages qui les énoncent.

\section{Du personnage au personnage-guide}

9 Si la définition du concept de personnage (d'autant plus lorsque celle-ci s'applique au domaine du jeu vidéo) pourrait à elle seule faire l'objet de longues discussions (à ce 
sujet, voir, entre bien d'autres, les travaux de Jouve, 1992, ou, en game studies, ceux de Blom, 2019 et 2020), nous utiliserons ici comme définition de travail, afin de servir efficacement les objectifs de cette recherche, celle proposée par Fotis Jannidis dans le living handbook of narratology, à savoir: "Le personnage est une figure textuelle ou médiatique au sein d'un monde fictionnel, généralement humaine ou semblable à l'humain ${ }^{8} »$ (Jannidis, 2013).

Cette définition attribue au personnage trois composantes fondamentales. Premièrement, ce dernier est pourvu d'un signifiant médiatique (il est «text- or mediabased ») : il appartient donc au domaine de la représentation, voire à celui de la fiction ("storyworld»), par opposition aux "personnes», qui évoluent dans le monde empirique (Jannidis, 2013) ${ }^{9}$. Deuxièmement, les personnages sont pris dans un récit, ou, du moins, dans un univers fictionnel (ils sont d'ailleurs souvent considérés comme les socles de la narrativité d'un texte médiatique, comme les éléments proéminents du récit, narratifs par excellence ${ }^{10}$ ). Troisièmement, les personnages sont des entités reconnues comme étant "semblables à l'humain» (human-like). Autrement dit, un signifiant devient un personnage dès le moment où on lui prête une certaine humanité, où l'interprète peut lui imaginer une vie propre, autonome, qui ne se limite pas à ses différentes apparitions médiatiques. Or nous tâcherons de montrer, à l'aide d'une série d'exemples, que les diverses incarnations du tutoriel qu'il est possible de rencontrer dans les jeux vidéo brouillent considérablement les frontières posées par cette définition.

\section{Deux niveaux d'existence : personnages interfaciels et avatars tutoriels}

11 Pour commencer, les personnages-guides compliquent le critère du signifiant médiatique, décrit ci-dessus, en ce que ces entités ne sont pas toujours dotées d'une forme identifiable et qu'il est parfois malaisé de les distinguer formellement de l'interface. Pour cette raison, il nous semble utile d'opérer une première distinction entre deux types de personnifications du tutoriel: les personnages interfaciels et les avatars tutoriels.

\section{Personnages interfaciels}

La première catégorie concerne les entités se situant à la frontière entre l'interface et le personnage-guide : elle rassemble les menus dotés d'une certaine "personnalité », les discours explicatifs pourvus d'une "voix narrative» qui les distingue des textes neutres du système, sans pour autant permettre de les associer à un personnage totalement identifiable. Un exemple parlant de personnage interfaciel peut être trouvé dans le jeu de plateformes The Addams Family: Pugsley's Scavenger Hunt (Ocean Software, 1993), sur NES. Dans ce dernier, le joueur peut en effet rencontrer des blocs rouges qui, s'il leur donne un coup de tête, délivreront un indice sous la forme d'un cadre contenant un texte et mettant le jeu en pause (voir Figure 2). 


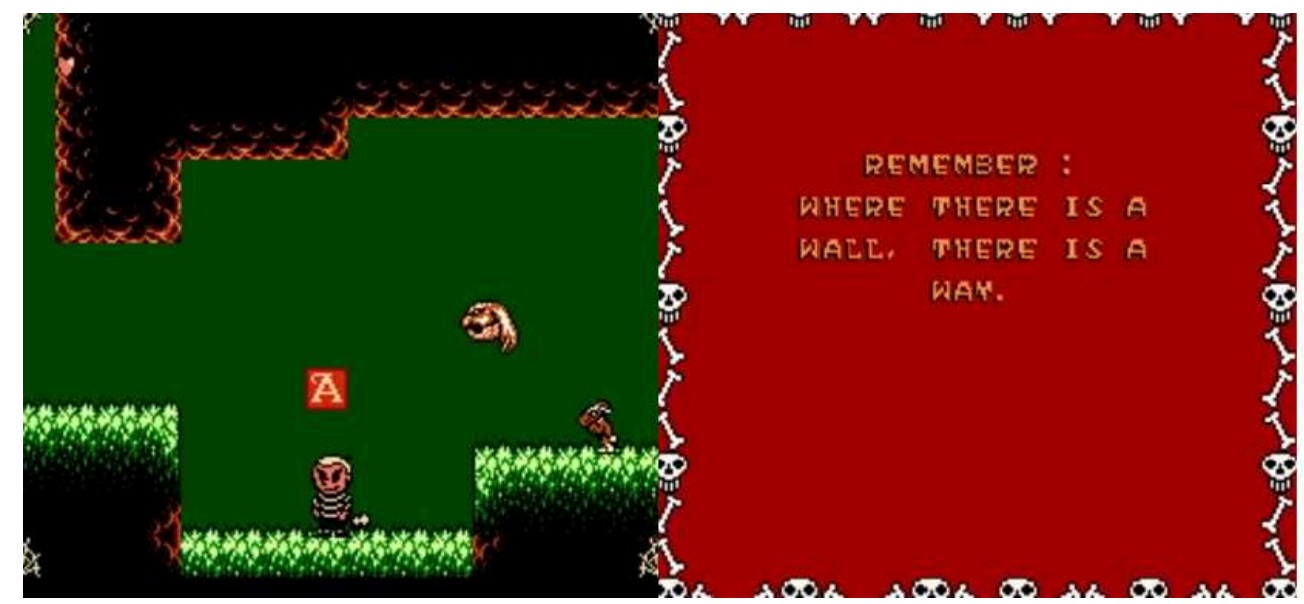

Figure (Tous Droits Réservés) - Blocs délivrant des indices dans The Addams Family: Pugsley's Scavenger Hunt

Bien que possédant une certaine tonalité humoristique ou proverbiale, ces textes ne sont pas attribués à un locuteur particulier au sein du jeu, si bien que le joueur est légitimement invité à les interpréter comme des indications extradiégétiques, prises en charge par le système et venant interrompre le cours de la partie comme de la narration. Cependant, la troisième page du manuel de ce jeu (manuel qui, comme pour la plupart des jeux NES de l'époque, contient la majeure partie des informations tutorielles) apprend à son éventuel lecteur que les textes d'aide sont en réalité prononcés par La Chose, l'un des personnages de la série La Famille Addams, qui a l'apparence d'une main humaine se déplaçant de façon autonome (voir Figure 3).

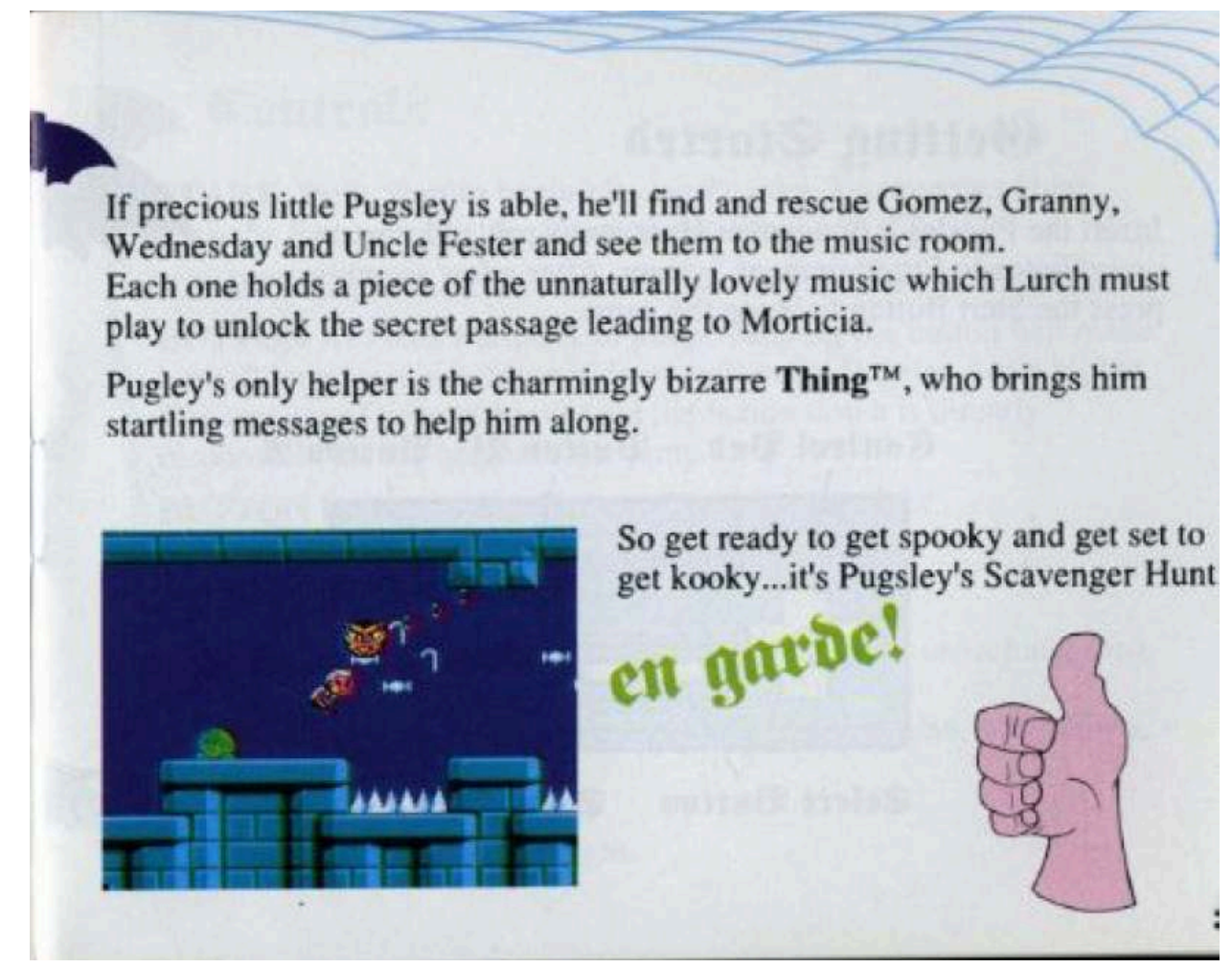

Figure (Tous Droits Réservés) - Troisième page du manuel de The Addams Family: Pugsley's Scavenger Hunt sur NES 
14 Au sein du jeu, pourtant, La Chose n'est pas dotée de signifiant clair : elle n'est pas nommée, elle n'y apparaît pas graphiquement et les textes du tutoriel dont elle est censée être l'énonciatrice ne sont pas assumés par un «je » reconnaissable. Il s'agit ainsi d'un cas ambivalent de personnage n'existant que par l'intermédiaire du paratexte (le manuel) et de l'intertexte : même si le jeu ne donne aucune indication sur son identité, on peut prêter à La chose le statut de personnage à condition d'en connaître les autres développements médiatiques (dans la série télévisée, les différents films, etc.). Si ces éléments participent à « personnifier » les menus du jeu, l'absence de développement de La Chose au sein de la partie ne permet toutefois pas d'interpréter l'entité comme un personnage à part entière, doté d'une intériorité et d'une vie propre.

Un exemple similaire peut être trouvé dans de nombreux jeux en réalité virtuelle (tels que, entre autres : Beat Saber ${ }^{11}$, Five Nights at Freddy's: Help Wanted ${ }^{12}$, Aperture Hand Lab ${ }^{13}$, The Climb ${ }^{14}$, etc.). Dans ceux-ci, il n'est en effet pas rare que le tutoriel soit transmis par l'intermédiaire d'une voix de narrateur ou de narratrice. Ce dispositif a l'avantage de livrer des informations tout en laissant le joueur libre de porter son regard où il le souhaite, ce qui est particulièrement utile pour les jeux développés sur ce support.

Dans ces exemples, il est difficile de trancher sur la nature intra- ou extradiégétique des voix tutorielles, qui restent relativement désincarnées : le joueur n'est pas amené à voir qui les prononce et ne peut les rattacher à un personnage rencontré par ailleurs dans la diégèse. Néanmoins, le fait qu'il s'agisse de voix (et non uniquement de textes écrits) dote, malgré tout, leurs énonciateurs d'une certaine corporéité, voire d'une ébauche de personnalité, en fonction du ton adopté (plus ou moins autoritaire, moqueur, encourageant, mystérieux, etc.). Certains de ces énonciateurs gagnent, en outre, des traits de caractérisation par le biais d'un rapport intertextuel: la voix robotique qui donne les instructions au joueur dans Aperture Hand Lab est ainsi assimilable à celle de "l'Annonceur » des jeux Portal ${ }^{15}$ (une voix masculine qui se fait entendre lorsque les autres personnages jouant le rôle de tutoriels sont absents), tandis que l'identité des diverses voix d'aide qu'il est possible d'entendre dans Five Nights at Freddy's: Help Wanted (celle du « Phone Guy », notamment) sont l'objet d'interprétations ou de théories de la part des joueurs, qui sont alimentées peu à peu au fil des jeux de la série.

17 Pour qualifier les entités indéfinies que représentent ces divers exemples, il est utile de recourir à la distinction opérée par Go Ito entre les notions de kyara et kyarakutaa (Ito, 2005 : 109-111). Si le kyarakutaa (transcription japonaise du terme anglais character) équivaut au personnage plein (doté d'un corps et d'une personnalité, similaire à une personne réelle, et dont on peut supposer qu'il est pourvu d'une vie propre), son abréviation, kyara, est un concept plus abstrait servant à désigner des cas de " protopersonnages", susceptibles de devenir des kyarakutaa complets si l'on approfondit leurs traits de caractère. Le kyara - dont les exemples prototypiques sont les mascottes, particulièrement répandues au Japon - est donc un ensemble de traits caricaturaux, simplifiés, formant une entité reconnaissable (qui peut même avoir un nom), dotée d'une identité et d'une certaine autonomie: elle est indépendante de son support original et conserve une certaine cohérence lorsqu'elle voyage de texte en texte (ce qui permet au public de la reconnaître). Pour autant, le kyara n'est pas développé au point de posséder une histoire, une intériorité complexe, un mode de vie, à la manière des personnages proprement dits. 

proto-personnages: reconnaissables dans l'intertexte, parfois associées à un nom générique donné par les joueurs eux-mêmes («Phone Guy», "Annonceur»), elles aident à poser la frontière minimale de ce que peut être un personnage-guide. D'autres exemples de figuration du tutoriel poussent toutefois plus loin le développement narratif.

\section{Avatars tutoriels}

Lorsque les règles ou les objectifs du jeu ne sont plus expliqués par le biais d'un menu, d'une interface, ou de ces êtres ambigus que sont les personnages interfaciels, mais sont transmis à travers le point de vue d'un personnage à part entière qui prend la responsabilité de ces informations, nous parlerons alors d'avatars tutoriels. Ces personnages ont une forme et une identité définies: ils sont des manifestations diégétisées de l'explication des règles, des personnifications de celle-ci.

Néanmoins, malgré leur statut de personnages pleins (de kyarakutaa), ces figures invitent à interroger le deuxième critère de la définition mentionnée en première partie de ce travail, à savoir celui de l'appartenance du personnage à un monde fictionnel. Parce qu'ils ont pour fonction de transmettre au joueur un mode d'emploi de la fiction ludique, les avatars tutoriels sont en effet des êtres fictionnellement ambigus : leurs discours explicatifs sont bel et bien intégrés à la diégèse (puisqu'ils sont énoncés par l'un de ses habitants), mais ils s'adressent directement à un récepteur extradiégétique, à savoir le joueur (en déguisant parfois ce discours transgressif derrière des adresses à l'avatar ; voir Nélide-Mouniapin, 2005). Le tutoriel est ainsi un type de discours fondamentalement réflexif (le jeu discourt sur lui-même) et hybride : il mêle constamment (parfois au sein d'une même phrase) des termes renvoyant à la fiction et d'autres renvoyant au monde empirique ou aux gestes du joueur. Ainsi, dans cette instruction issue de Kirby's Adventure (HAL Laboratory, Nintendo, 1993), le bouton A et Kirby ne se situent pas sur le même plan d'existence: « Appuie sur le bouton A et Kirby saute ${ }^{16}$ !» (voir Figure 4). 


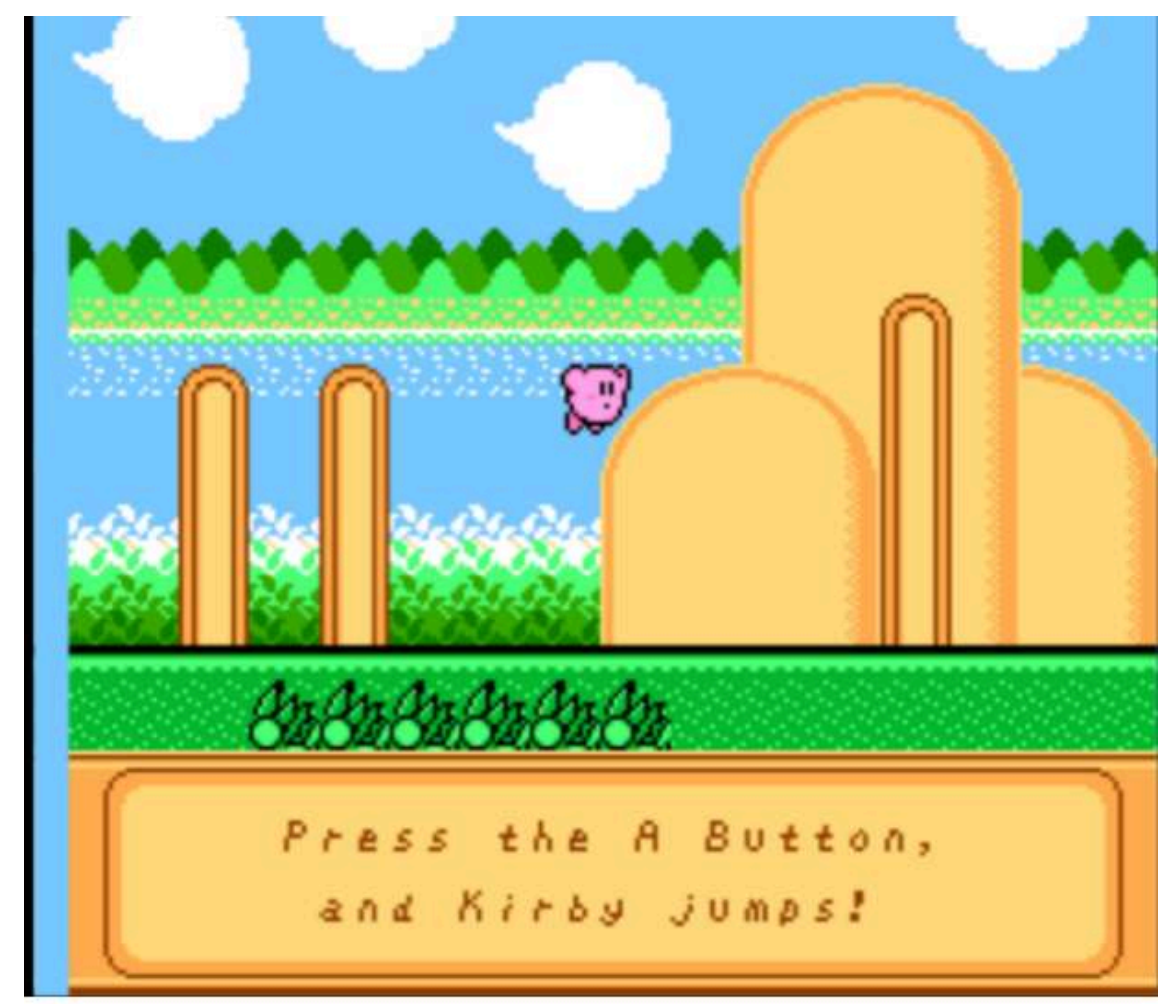

Figure (Tous Droits Réservés) - L'une des phrases du tutoriel de Kirby's Adventure sur NES

L'intégration de ces explications au sein de l'univers ludique implique, en d'autres termes, la production de nombreuses métalepses ${ }^{17}$ et mises en abyme - procédés métadiscursifs ainsi érigés, dans le domaine du jeu vidéo, en véritables conventions narratives (à ce sujet, voir Ryan, 2004 et Ensslin, 2015).

Or il est possible de distinguer trois principales formes d'avatarisation du tutoriel en fonction de la manière dont le personnage-guide gère la nature métaleptique des informations qu'il transmet: celui-ci peut tenter de dissimuler la transgression que sa prise de parole opère dans les frontières de la fiction, il peut la laisser apparente, ou encore la souligner. Ces trois stratégies rhétoriques seront examinées plus en détail cidessous, car elles ont un effet direct sur le statut conféré au personnage qui les emploie.

\section{Métaphores tutorielles}

La première stratégie rhétorique possiblement adoptée par les avatars tutoriels consiste à dissimuler la nature métaleptique de leur discours en intégrant celui-ci à la diégèse. Dans ce cas de figure, toutes les explications livrées par ces personnages sont formulées en des termes qui appartiennent au monde fictionnel, de sorte que ces phrases ne contiennent pas de rupture explicite. Les formules renvoyant au monde empirique, au système de jeu, à ses supports ou périphériques sont ainsi " traduites " par le biais de métaphores. La «mort » de l'avatar, par exemple, permet bien souvent de désigner l'échec du joueur; les «vies» ou la «santé» décrivent indirectement les crédits ou le nombre de tentatives dont il dispose; etc. Ce procédé de transcription peut également passer par le biais d'un habillage graphique, comme dans les nombreux exemples de menus "diégétisés». Dans certains jeux en réalité virtuelle tels que Arizona Sunshine (Vertigo Games, 2016), Superhot VR (Superhot Team, 2016) ou Job 
Simulator: The 2050 Archives (Owlchemy Labs, 2016), pour lancer un niveau, le joueur devra ainsi métaphoriquement insérer une cassette dans un lecteur ou une disquette dans un ordinateur.

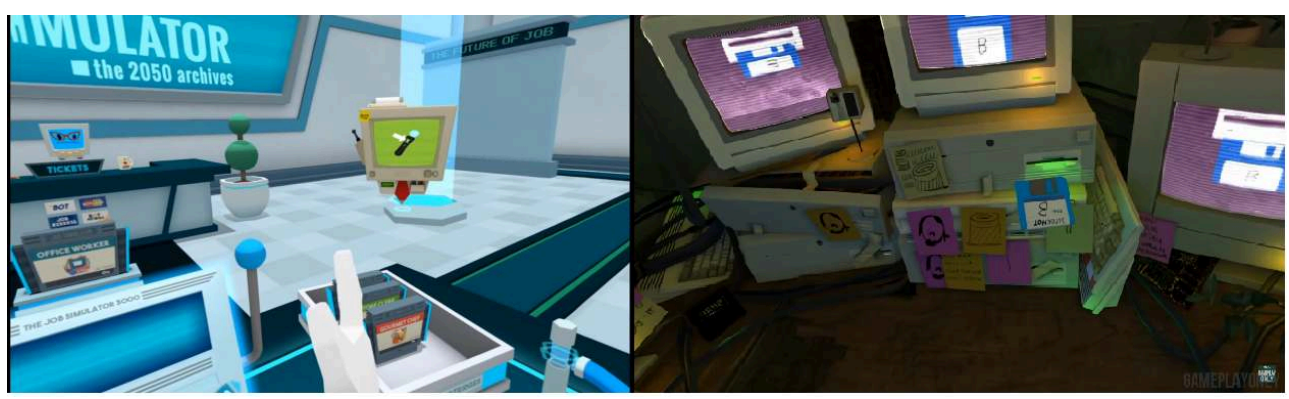

Figure (Tous Droits Réservés) - Menu de lancement du jeu dans Job Simulator: The 2050 Archives (à gauche) et Superhot VR (à droite)

Pour illustration, dans le jeu d'aventure-action Okami (Clover Studio, Capcom, 2006), le joueur est guidé dans son aventure par un personnage nommé Issun, dont les commentaires visent à éclairer non seulement les développements de l'histoire, mais aussi les objectifs du jeu, les contrôles et les mécaniques de gameplay. Dans l'un des niveaux, Issun donne à l'avatar Amaterasu (et, par son intermédiaire, au joueur) les conseils suivants: "Je suis là pour t'aider quand tu en as vraiment besoin. / Par exemple, tu vois ce coffre tout enchaîné là-bas? Si tu peux me guider vers lui avec ton Pinceau Céleste, / je ferai un saut dessus et je volerai ce qu'il y a dedans ${ }^{18}$ ! (voir Figure 6).

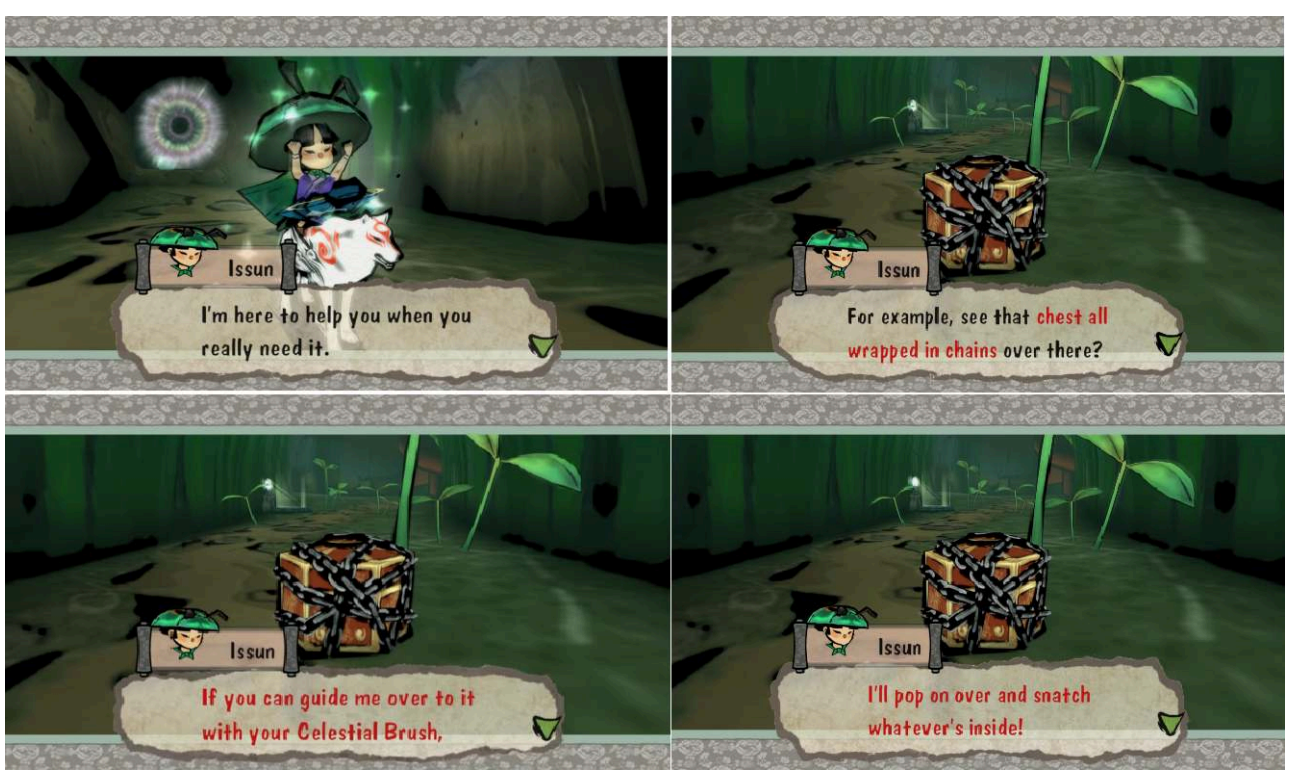

Figure (Tous Droits Réservés) - Dialogues d'aide prononcés par Issun, l'avatar tutoriel du jeu Okami

Ce texte ne contient que des termes qui font sens au sein de l'univers de cette œuvre, mais donne au joueur des instructions précises sur les gestes qu'il est censé effectuer et sur les boutons sur lesquels il est censé appuyer. Le "Pinceau Céleste » est ainsi une métaphore mise en place par le jeu pour désigner une mécanique de gameplay (la possibilité de tracer des formes à l'écran en maintenant les boutons «R1 » et "carré " de la manette de Playstation) comme s'il s'agissait d'un attribut divin de la protagoniste. L'action de "guider " Issun vers le coffre est également une manière détournée de signaler au joueur qu'il doit dessiner un trait entre le coffre et le PNJ. 
La plupart des jeux vidéo standardisent ainsi, dès le début de la partie et par le biais de leurs personnages, un répertoire métaphorique dans lequel ils puiseront de façon répétée pour donner des directives à l'utilisateur sans énoncer de métalepse brute. Il est néanmoins intéressant de noter que, dans l'exemple mentionné ci-dessus, les termes véhiculant des informations utilitaires sont soulignés par une couleur différente (ils apparaissent en rouge pour marquer leur importance). Dans Okami (comme dans d'autres titres), la matérialité du texte met ainsi en avant le fait que certains énoncés n'ont pas tout à fait le même statut que les autres discours diégétiques: sans être explicitement métaleptiques, toutes les formules en rouge contiennent ici, en creux, une injonction à agir ; en bons textes injonctifs, elles annoncent métonymiquement une nécessaire future action du joueur.

\section{Métalepses brutes}

La seconde stratégie rhétorique par laquelle les avatars tutoriels peuvent transmettre leurs conseils et directives est celle, déjà évoquée en guise d'introduction, consistant à entremêler les informations fictionnelles et empiriques dans le discours, sans adoucir la friction entre ces deux niveaux ontologiques. Dans cette catégorie, les personnages ne tentent donc pas de traduire les réalités empiriques en termes diégétiques, mais ne soulignent pas non plus les ruptures qu'ils opèrent : celles-ci sont énoncées de manière directe et non problématisée, sans être encadrées par de quelconques marqueurs qui signaleraient leur statut discursif particulier, si bien qu'elles peuvent être qualifiées de métalepses brutes.

Dans Captain America and The Avengers (Data East, 1991) sur NES, par exemple, l'avatar du joueur est guidé par les instructions de sa collègue super-héroïne, la Guêpe (Wasp). Or celle-ci énonce régulièrement des formules métaleptiques, telles que : «Cap évitera l'eau et les pics en sautant et en appuyant sur la flèche du bas ${ }^{19}{ }$. Ce type de formule s'adresse assez directement au joueur (l'avatar, Captain America, n'est mentionné qu'à la troisième personne, comme s'il n'était pas concerné par le message) et réfère à des objets empiriques (les boutons de la manette) dont les personnages fictionnels ne sont pas censés connaître l'existence. Cependant, ces dialogues n'en sont pas moins énoncés par l'un de ces personnages et entendus par l'avatar. Celui-ci répond d'ailleurs systématiquement à ces informations absconses, s'adressant à un tiers et portant sur un monde qu'il ne connaît pas, par un remerciement ou un sobre: "Compris, la Guêpe $^{20} »$ (voir Figure 7).

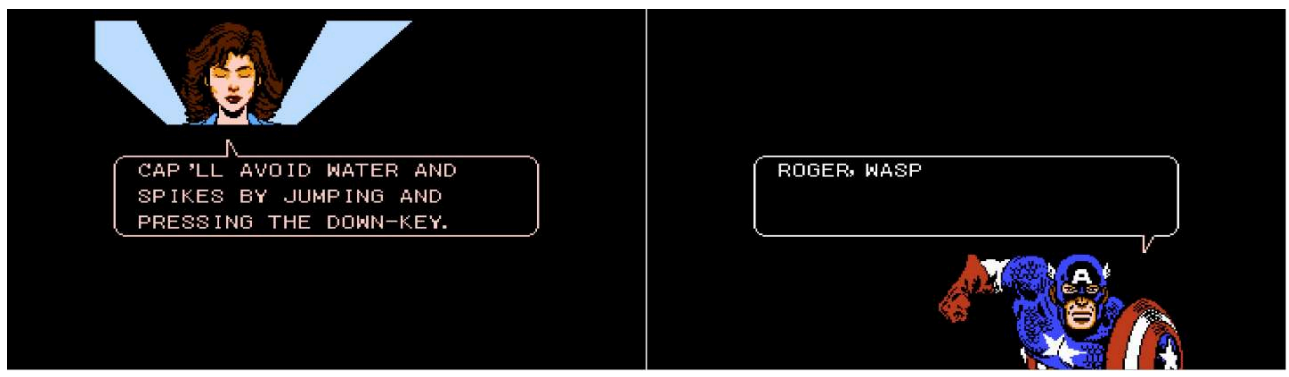

Figure (Tous Droits Réservés) - Intervention de l'avatar tutoriel et réponse de l'avatar dans Captain America and The Avengers

29 Pour citer un exemple similaire issu d'un titre plus récent, l'on peut mentionner le jeu de coopération Overcooked (Ghost Town Games, Team17, 2016). Le Roi Oignon (avatar tutoriel et destinateur de la quête principale) mentionne effectivement dans ses 
explications des éléments extérieurs à sa diégèse, tels que les boutons ou l'écran, tout en les associant à des impératifs narratifs : «Vite! Prenez un ingrédient avec le bouton [Espace]!»; «C'est bien! Suivez les commandes en haut de l'écran. Nous finirons par vaincre cette Bête !» (voir Figure 8).

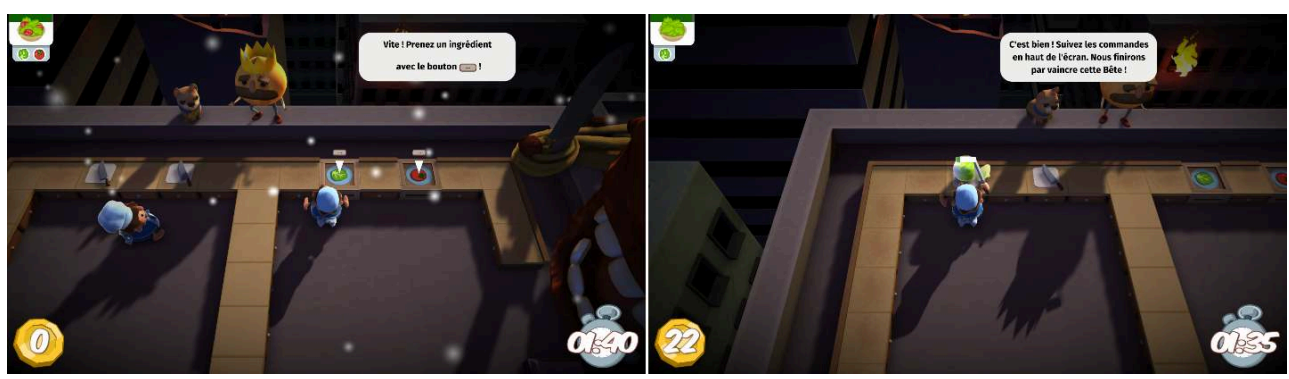

Figure (Tous Droits Réservés) - Tutoriel d'Overcooked

Malgré leur incohérence narrative, les illustrations de ces types de discours dans les jeux sont nombreuses au point d'en être conventionnelles. C'est notamment pour cette raison qu'Astrid Ensslin considère les jeux vidéo comme des récits " non naturels par excellence » (Ensslin, 2015: 43) : loin de représenter le monde de façon mimétique, les fictions ludiques déconstruisent constamment les codes classiques de la narration, parmi lesquels la fermeture et la cohérence des univers fictionnels. Les métalepses brutes observables dans les tutoriels ne peuvent donc être considérées comme des figures véritablement transgressives dans le contexte particulier du jeu vidéo (Ryan, 2004 ; Allain, 2018), puisqu'elles relèvent plutôt d'une convention ludo-narrative, présupposée par l'articulation entre l'activité du joueur et la fiction. Néanmoins, si elles ne constituent pas des ruptures, ces métalepses participent à conférer aux avatars tutoriels un statut particulier: par la «conscience» qu'ils manifestent du monde empirique, ces êtres fictionnels ne se situent pas tout à fait sur le même plan que les autres habitants de la diégèse.

\section{Métalepses fictionnalisées}

31 Enfin, une troisième stratégie, plus rare, mais aux effets rhétoriques plus marqués, peut être utilisée par les avatars tutoriels pour convier des informations : ceux-ci peuvent souligner l'existence d'une métalepse dans leur discours et montrer qu'ils sont conscients d'opérer une transgression des seuils de la fiction.

Un exemple souvent commenté est celui du personnage de Monika dans le visual novel Doki Doki Literature Club! (Team Salvato, 2017). Au début du jeu, la présidente du club de littérature profite effectivement de l'un des "conseils d'écriture" qu'elle livre à l'avatar pour introduire le joueur au système de sauvegarde (et pour lui laisser entendre que le jeu constituera une narration à embranchements au sein de laquelle il devra faire des choix de parcours): "Parfois, tu te trouveras face à une décision difficile... Lorsque cela se produit, n'oublie pas de sauvegarder ta partie! Tu ne sais jamais quand tu pourrais changer d'avis... ...ou quand quelque chose d'inattendu pourrait se produire!». Immédiatement après avoir fait ce commentaire, elle s'arrête toutefois pour en souligner l'incongruité : «Attends... ce conseil concerne-t-il vraiment l'écriture ? De quoi est-ce que je suis en train de parler? Ahaha! ...C'est mon conseil pour aujourd'hui' ${ }^{21}$ !» (voir Figure 9). 


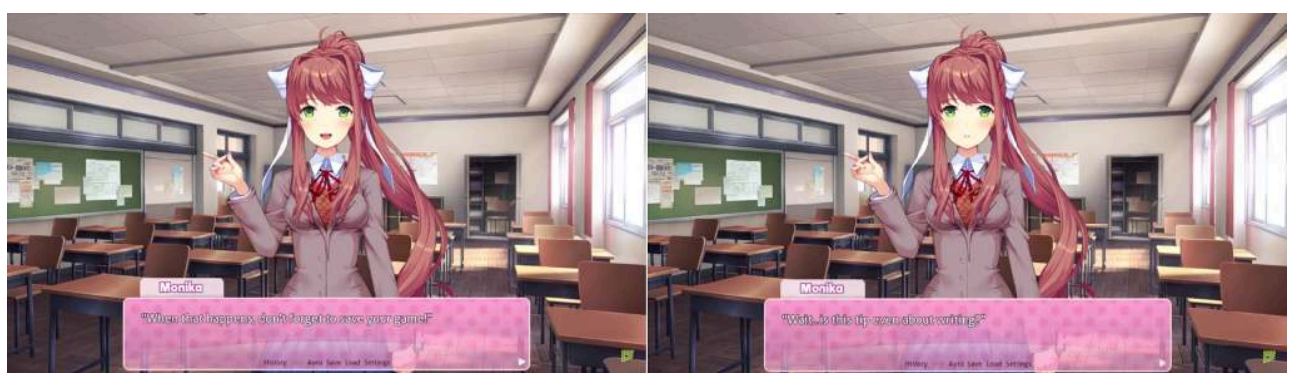

Figure (Tous Droits Réservés) - Monika manifeste être consciente de la métalepse qu'elle opère dans Doki Doki Literature Club!

Si la mention de la sauvegarde par un personnage serait passée inaperçue dans un autre jeu (en raison de la nature conventionnelle des métalepses brutes décrite cidessus), la rupture est ici montrée du doigt et utilisée comme mécanisme narratif : plus loin dans le jeu, Monika révèlera effectivement avoir conscience de sa nature fictionnelle. L'écart opéré lors du tutoriel constituait en réalité un premier indice de la monstruosité de ce personnage, annonçant le basculement générique du jeu depuis la simulation de drague vers l'horreur psychologique.

De nombreuses illustrations de ce procédé peuvent également être trouvées dans le jeu de rôle japonais EarthBound (Ape et HAL Laboratory, Nintendo, 1994), connu au Japon sous le titre de Mother 2: Gyiyg Strikes Back. L'un des premiers avatars du tutoriel de ce jeu, Pokey, va ainsi commencer par s'excuser auprès du joueur de ne pas avoir traduit son explication en termes fictionnels : "Désolé de te donner ce conseil typique de jeu vidéo, mais tu devrais équiper ton arme ${ }^{22}$ ! $»$. Plus loin, il suggère qu'il est conscient de se trouver dans un jeu: "[La fonction] "Équiper" est beaucoup utilisée dans les jeux comme celui-ci, mais ça tu le savais déjà ${ }^{23} \ldots$ ».

Plusieurs autres personnages, tels que les "Taupes d'assistance", ont également tendance à se corriger de façon humoristique à chaque fois qu'ils emploient, dans leurs messages informatifs, des termes extradiétiques. Lorsqu'elle expose au joueur le comportement des ennemis et le fonctionnement des combats, la première Taupe d'assistance se reprend de cette façon: «Quand ils [les ennemis] t'approchent de cette manière, ton écran de TV, non... non... / Je veux dire que le monde devant toi va montrer un tourbillon rouge pendant une seconde ${ }^{24} »$. Dans la suite de son explication, elle répète son simulacre de maladresse : "Dans ce cas-là, ta télévision... Oups je l'ai encore fait ${ }^{25}$ !». Une autre Taupe du tutoriel, plus loin dans l'aventure, entrecoupe ses explications de plaisanteries similaires: "C'est dû à l'action en cours de ta jauge de $\mathrm{PV}^{26}$. Oups ! Je veux dire ta vie ${ }^{27} »$. 


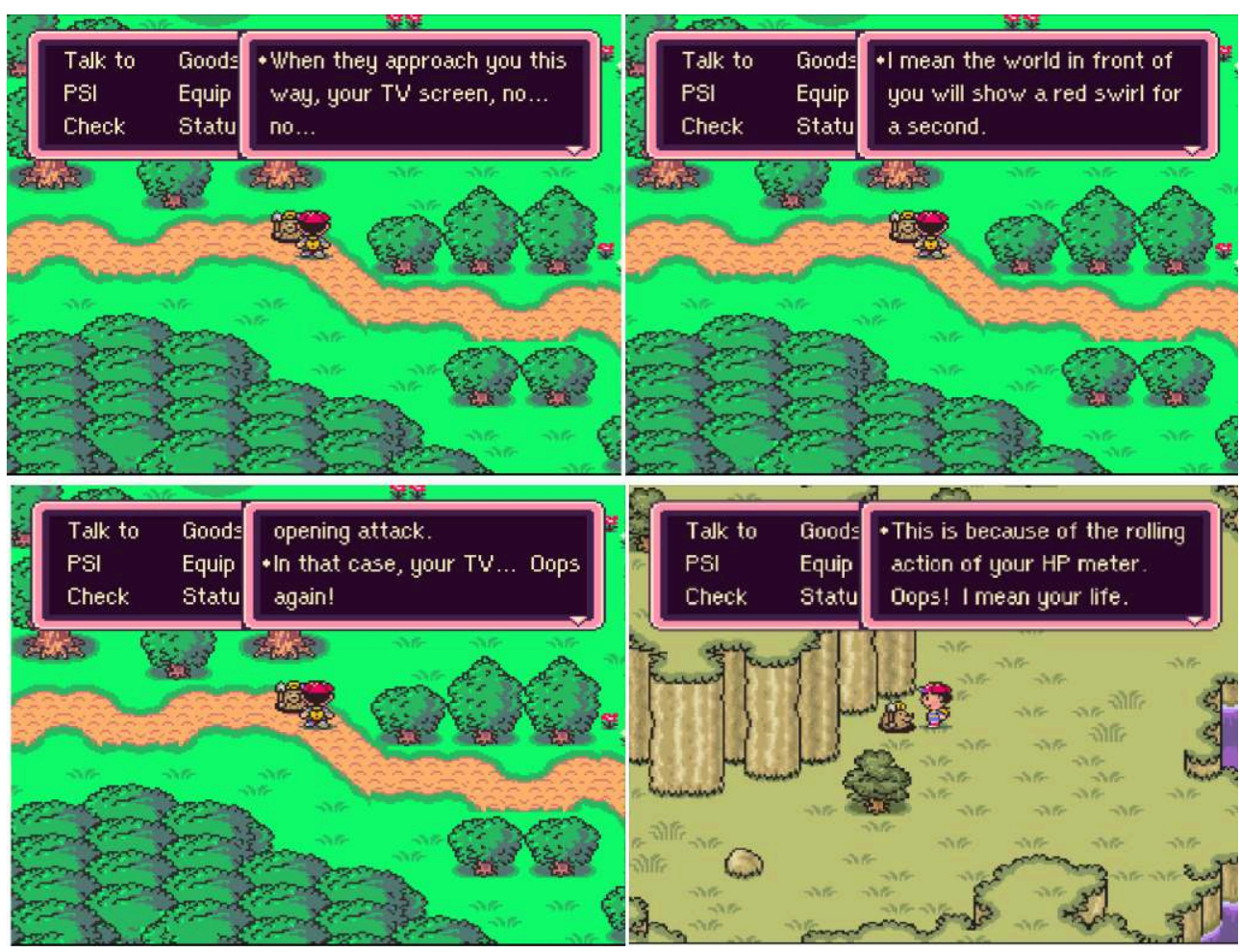

Figure (Tous Droits Réservés) - Les Taupes d'assistance d'EarthBound se reprennent

humoristiquement lorsqu'elles emploient un terme qui ne renvoie pas à la diégèse

En assumant explicitement les métalepses qu'ils déclarent, en soulignant la friction qui existe entre les parts diégétiques de leur discours et celles qui renvoient au monde empirique, ces personnages réintègrent d'une certaine manière les métalepses tutorielles au sein de l'univers fictionnel. Ce faisant, ils soulignent la nature conventionnelle des métalepses dans les jeux vidéo; ils mettent au jour le caractère fondamentalement métaleptique, hybride, des fictions vidéoludiques. En d'autres termes, dans les jeux qui utilisent ce procédé, les discours tutoriels font partie de la diégèse en tant que métalepses, puisqu'ils sont reconnus comme tels par les personnages qui la peuplent. Pour cette raison, nous proposons d'appeler ces procédés rhétoriques des métalepses fictionnalisées: loin d'interrompre la narration, elles sont intégrées dans son développement et, à travers elles, une partie du monde empirique est aussi incorporée dans l'univers fictif.

L'effet de transgression ne provient donc pas vraiment, ici, de la rupture d'un seuil de la fiction (puisqu'elle est attendue), mais de la mise en évidence de cette rupture : les $\mathrm{PNJ}$ se distancient de leur univers et de ses règles en commentant ironiquement leur artificialité (le monde n'est qu'un " écran de TV », la vie n'est qu'une " jauge », etc.). Le fait qu'ils se corrigent d'eux-mêmes suggère par ailleurs que, dans le reste de leurs prises de parole, ils prétendent ne pas être conscients que leur monde est fictif : ils font comme s'ils ne savaient pas qu'ils se trouvaient dans un jeu vidéo.

38 Cette attitude distanciée n'est pas sans rappeler les définitions qui ont été données du jeu ou de l'attitude ludique, notamment par Henriot (1989 : 300), Schaeffer (1999: 234) et Genvo (2013). Ces derniers auteurs décrivent l'attitude du joueur comme un acte de prise de distance par rapport au monde environnant, comme un "procès métaphorique » (Henriot, 1989: 300) par lequel le joueur agit «comme si » ce qu'il faisait était autre (Genvo, 2013). Ainsi, les avatars tutoriels décrits ci-dessus sont 
construits comme des figures de joueurs, adoptant un simulacre d'attitude ludique visà-vis de leur propre monde. Le tutoriel qu'ils délivrent n'est donc pas seulement composé d'instructions sur les règles et le gameplay: il s'agit aussi d'un mode d'emploi de l'attitude qui est attendue du joueur. À travers ces discours, le jeu invite le joueur à imiter la réflexivité des personnages, à prendre du recul par rapport au système ludique, à remettre en question sa logique et à reprendre conscience du caractère arbitraire et artificiel des conventions qui le dirigent.

\section{Conclusion: les avatars de la machine, un nouvel archétype de personnage?}

Partant de la définition donnée par Jannidis (2013) du concept de personnage, nous avons proposé, dans cet article, de nous pencher sur le cas particulier des «personnages-guides » vidéoludiques, ces incarnations narrativisées du tutoriel dans les jeux. En les examinant d'un point de vue narratologique (comment participent-ils à la narration?) et rhétorique (quelles sont les stratégies discursives qu'ils emploient pour transmettre leurs instructions?), il est apparu que ces entités invitent à interroger les critères principaux servant d'assise au concept de personnage. D'une part, le signifiant médiatique de ces êtres n'est pas toujours clair: les tutoriels personnifiés n'ont pas nécessairement une forme identifiable, se confondent partiellement avec l'interface et se limitent donc parfois à l'étape du kyara (Ito, 2005), du proto-personnage. D'autre part, l'inclusion des personnages-guides à l'univers fictionnel est aussi à nuancer : par les constantes ruptures qu'ils opèrent dans les frontières diégétiques, les avatars tutoriels ne sont pas tout à fait situés sur le même plan d'existence que les autres PNJ, mais évoluent aux marges de la fiction.

Dans le prolongement de cette réflexion, nous souhaiterions, enfin, revenir sur le troisième critère définitoire du personnage mentionné en introduction, à savoir le fait qu'il serait construit à l'image de l'humain. Cette réflexion permettra, dans le même temps, de préciser pourquoi nous avons jusqu'ici choisi les termes d'avatar ou d'avatarisation pour qualifier les PNJ pourvoyeurs d'informations, et non ceux de personnification ou d'incarnation du tutoriel dans un personnage. Par ce choix, nous avons en effet souhaité marquer le fait que l'hybridité ontologique des personnagestutoriels les différencie considérablement des autres PNJ qu'il est possible de rencontrer dans un jeu vidéo et les rapproche plutôt du fonctionnement de l'avatar.

41 En tant que médiation entre le joueur et l'objet-jeu, l'avatar est ce que Genette appelle un « opérateur de métalepse » $(2004: 110)$ : il représente à la fois un élément fictionnel (un habitant du monde représenté) et un élément empirique (le geste du joueur) au sein du jeu (Barnabé et Delbouille, 2018). Or, de la même manière, les personnages-guides représentent un contenu empirique (les règles, les contrôles) à travers une prothèse fictive (le personnage). En opérant ce transfert, ils deviennent les avatars, non du joueur, mais de la machine. Ces entités particulières sont ainsi des êtres partiellement empiriques et partiellement fictionnels: dialoguer avec eux, c'est dialoguer avec le système du jeu. Par leur caractère fondamentalement métaleptique, ces personnages sont, d'une certaine manière, construits comme les égaux de l'avatar du joueur, ce qui a pour conséquence de faire perdre à ce dernier son statut d'exceptionnalité dans l'univers vidéoludique : le joueur n'est pas la seule entité empirique à être représentée de manière fictionnalisée au sein du jeu. 

seulement l'humain. Pour conclure sur ce point, repartons de la citation suivante de John Frow au sujet du rapport entre personnages et personnes :

Notre reconnaissance du type de choses que sont les personnages de fiction dépend de notre connaissance préalable du type de choses que sont les personnes. Nous comprenons les personnages comme des quasi-personnes. Mais la modélisation va également dans l'autre sens: notre compréhension des personnes est, en partie, façonnée par notre expérience des personnages de fiction ${ }^{28}$ (Frow, $2014: 107$ ).

43

En d'autres mots, traditionnellement, un signe médiatique est reconnu comme un personnage parce qu'on lui prête des traits humanisés (et le fait de côtoyer de nombreux personnages alimente et détermine la façon dont nous concevons ce que recouvre ce procédé d'humanisation). Toutefois, l'hypothèse que nous souhaiterions formuler est qu'à travers les personnages-guides, les incarnations narrativisées des tutoriels, un nouvel archétype de personnage est peut-être en train de se former : un type d'entité à qui l'on reconnaît le statut de personnage autonome, doté d'une identité et d'une existence propre, non parce qu'il est semblable aux humains, mais parce qu'il ressemble aux machines. Les nombreuses figures de robots ou d'intelligences artificielles qui sont apparues ces dernières années dans les œuvres ludiques pour guider le joueur tout en mettant en abyme le système de jeu (dont GLaDOS, de la série Portal, est sans doute l'exemple le plus emblématique) illustrent la solidification progressive de cet archétype.

Or, comme le notait Frow ci-dessus, si les avatars tutoriels témoignent d'une évolution dans ce que nous reconnaissons comme des personnages, cette évolution n'est peutêtre pas sans lien avec les mutations dans la manière dont nous nous concevons en tant que personnes. Cette hypothèse déborde du cadre du présent article pour entrer dans le champ des questions liées au posthumanisme (voir Guesse, 2019) et mériterait un examen approfondi dans ce cadre. Néanmoins, elle nous sert ici à mettre au jour l'intérêt d'étudier plus avant les tutoriels vidéoludiques en dépassant les seules questions de design et d'ergonomie, pour reconnaître la centralité de ces dispositifs au sein du médium vidéoludique et le prisme précieux qu'ils constituent pour interroger ses possibilités narratives.

\section{BIBLIOGRAPHY}

Allain, Sébastien (2018), « Métalepses du récit vidéoludique et reviviscence du sentiment de transgression », Sciences du jeu, $n^{\circ}$ 9, en ligne, consulté le 21 juillet 2020, URL : https:// journals.openedition.org/sdj/909

Andersen, Erik et al. (2012), « The Impact of Tutorials on Games of Varying Complexity », Proceedings of the SIGCHI Conference on Human Factors in Computing Systems CHI'12, Austin, ACM, pp. 59-68, en ligne, consulté le 21 juillet 2020, URL : http://grail.cs.washington.edu/ projects/game-abtesting/chi2012/chi2012.pdf

Cahiers de Narratologie, 38 | 2020 
Barnabé, Fanny (2019), « Narratological and Rhetorical Functions of Video Game Tutorials: The case of NES Games », Communication à Replaying Japan 2019 - Japanese Games: Past, Present and Future, Kyoto, en ligne, consulté le 21 juillet 2020, URL : https://orbi.uliege.be/handle/ $2268 / 238910$

Barnabé, Fanny et Delbouille, Julie (2018), « Aux frontières de la fiction : l'avatar comme opérateur de réflexivité », Sciences du jeu, $n^{\circ}$ 9, en ligne, consulté le 21 juillet 2020, URL : https:// journals.openedition.org/sdj/958

Blom, Joleen (2019), « A Ludo Mix Perspective on Dynamic Game Characters », Abstract Proceedings of the 2019 DiGRA International Conference: Game, Play and the Emerging Ludo-Mix, en ligne, consulté le 21 juillet 2020, URL : http://www.digra.org/wp-content/uploads/digitallibrary/DiGRA_2019_paper_49.pdf

Blom, Joleen (2020), The Dynamic Game Character: Definition, Construction, and Challenges in Character Ecology, Thèse de doctorat en Game Studies, IT University of Copenhagen

Ensslin, Astrid (2015), « Video Games as Unnatural Narratives », in Diversity of Play, M. Fuchs (dir.), Lunebourg, Meson Press, pp. 41-72

Frommel, Julian et al. (2017), « The Effects of Context-Sensitive Tutorials in Virtual Reality Games ", Proceedings of the 2017 Annual Symposium on Computer-Human Interaction in Play CHI PLAY'17, Amsterdam, ACM, pp. 367-375, en ligne, consulté le 21 juillet 2020, URL : https:// dl.acm.org/doi/pdf/10.1145/3116595.3116610

Frow, John (2014), Characters and Person, Oxford, Oxford University Press

Genette, Gérard (1982), Palimpsestes. La littérature au second degré, Paris, Seuil

Genette, Gérard (2004), Métalepse : de la figure à la fiction, Paris, Seuil

Genvo, Sébastien (2008), « Du rôle de la masculinité militarisée dans la médiation ludique sur support numérique », Quaderni, $\mathrm{n}^{\circ}$ 67, pp. 43-52, en ligne, consulté le 21 juillet 2020, URL : https://journals.openedition.org/quaderni/282

Genvo, Sébastien (2013), « Penser les phénomènes de ludicisation à partir de Jacques Henriot ", Sciences du jeu, $\mathrm{n}^{\circ} 1$, en ligne, consulté le 21 juillet 2020, URL : http://journals.openedition.org/ $\operatorname{sdj} / 251$

Guesse, Carole (2019), Fictions and Theories of the Posthuman: From Creature to Concept, Thèse de doctorat en Langues, Lettres et Traductologie, Université de Liège, en ligne, consulté le 21 juillet 2020, URL : https://orbi.uliege.be/bitstream/2268/241983/1/Thesis_CaroleGuesse.pdf Henriot, Jacques (1989), Sous couleur de jouer : la métaphore ludique, Paris, José Corti Ito, Go (2005), Tezuka izu deddo: Hirakareta manga no hyōgenron e, Tōkyō, NTT Shuppan Jannidis, Fotis (2013), « Character », in the living handbook of narratology, P. Hühn et al. (dirs.), Hamburg, Hamburg University, en ligne, consulté le 21 juillet 2020, URL : https://www.lhn.unihamburg.de/node/41.html Jouve, Vincent (1992), L'effet-personnage dans le roman, Paris, Presses Universitaires de France Koster, Raph (2013), Theory of Fun for Game Design, Sebastopol, O’Reilly Media

Morin, Raphaël et al. (2016), « The Effect of Game Tutorial: A comparison between casual and hardcore gamers ", Proceedings of the 2016 Annual Symposium on Computer-Human Interaction in Play CHI PLAY Companion'16, Austin, ACM, pp. 229-237, en ligne, consulté le 21 juillet 2020, URL : https://dl.acm.org/doi/pdf/10.1145/2968120.2987730 
Nélide-Mouniapin, Bernadette (2005), « Exemple d'énonciation

énonciation

dans un jeu vidéo ", in Le game design de jeux vidéo. Approches de l'expression vidéoludique, S. Genvo (dir.), Paris, L’Harmattan, pp. 239-251

Raffaele, Rennan Cavalcante, de Carvalho, Breno José Andrade et Silva, Frutuoso G. M. (2017), «Evaluation of immersive user interfaces in virtual reality first person games », Communication à EPCGI 2017, Guimarães, en ligne, consulté le 21 juillet 2020, URL : https:// pdfs.semanticscholar.org/e1b5/5f73a1037181be6399b4e9a1aef20ba50205.pdf? _ga=2.32182974.1895087919.1595428532-1860513494.1581174033

Ryan, Marie-Laure (2004), « Metaleptic Machines », Semiotica, n 150, pp. 439-469

Schaeffer, Jean-Marie (1999), Pourquoi la fiction?, Paris, Seuil

Schell, Jesse (2008), The Art of Game Design: A Book of Lenses, Burlington, Morgan Kaufmann Steinkuehler, Constance et al. (dirs..) (2012), Games, Learning, and Society: Learning and Meaning in the Digital Age, Cambridge, Cambridge University Press

Therrien, Carl et Julien, Mikaël (2015), « "Pour obtenir de l'aide, appuyez sur X”. La montée du paradigme d'assistance dans le design du jeu vidéo », Sciences du jeu, $\mathrm{n}^{\circ} 4$, en ligne, consulté le 21 juillet 2020, URL : https://journals.openedition.org/sdj/508

\section{NOTES}

1. Pour plus d'informations sur la constitution de cette base de données, voir Barnabé (2019).

2. Role-Playing Game, ou jeu de rôle.

3. Acrony de « Personnage Non Joueur».

4. « Use keys in palaces they are found in ». Toutes les traductions de l'anglais présentes dans cet article sont personnelles.

5. « Do not go South without a candle».

6. « When you jump press downward to stab».

7. « Sorry. I know nothing».

8. "Character is a text- or media-based figure in a storyworld, usually human or human-like ».

9. Bien que cette distinction soit évidemment loin d'être absolue.

10. «Pour la plupart des lecteurs, les personnages constituent l'un des aspects les plus importants d'un récit », (« For most readers, characters are one of the most important aspects of a narrative »; Jannidis, 2013).

11. Beat Games, 2019.

12. Steel Wool Studios, ScottGames and Lionsgate Games, 2019.

13. Cloudhead Games, Valve Corporation, 2019.

14. Crytek, 2016.

15. Valve, 2007.

16. «Press the A Button, and Kirby jumps! ».

17. "J'entends par métalepse (Figures III, p. 243) toute espèce de transgression, surnaturelle ou ludique, d'un palier de fiction narrative ou dramatique, comme lorsqu'un auteur feint de s'introduire dans sa propre création, ou d'en extraire un de ses personnages » (Genette, 1982: 527).

18. «I'm here to help you when you really need it. / For example, see that chest all wrapped in chains over there? / If you can guide me over to it with your Celestial Brush, / I'll pop on over and snatch 
whatever's inside! ». Les passages en italique sont ceux qui, dans le jeu, apparaissent en rouge pour marquer qu'il s'agit d'informations utilitaires.

19. «Cap'll avoid water and spikes by jumping and pressing the down-key ».

20. « Roger, Wasp ».

21. "Sometimes you'll find yourself facing a difficult decision... When that happens, don't forget to save your game! You never know when you might change your mind... ..or when something unexpected may happen! Wait... is this tip even about writing? What am I even talking about? Ahaha! ...That's my advice for today!».

22. «Sorry about giving you this game-type advice, but you should equip your weapon!».

23. " "Equip" is used a lot in games like this, but you already knew that... ".

24. "When they approach you this way, your TV screen, no... no... / I mean the world in front of you will show a red swirl for a second ».

25. «In that case, your TV... Oops again! ».

26. Abréviation de «Points de Vie » et traduction de HP (qui est, en anglais, l'abréviation de « Health Points »).

27. "This is because of the rolling action of your HP meter. Oops! I mean your life ».

28. "Our recognition of the kind of thing fictional characters are depends on our prior knowledge of the kind of thing persons are. We understand characters as quasi-persons. But the modeling goes the other way as well: our understanding of persons is, in part, shaped by our experience of dealing with fictional characters ».

\section{ABSTRACTS}

This paper proposes to study video game tutorials from a narratological and rhetorical perspective. More precisely, it focuses on the case of the "guiding-characters", these narrative incarnations of the tutorial that accompany the avatar's progression by dictating to the player the steps to follow at each stage of the game. These hybrid beings represent an ideal entry point for studying the narrativization mechanisms of the rules at work within the medium, since they combine a utilitarian function (they reveal the game's instructions) and a narratological function (they advance the narrative). Through the analysis of a series of examples, this research will aim to demonstrate that guiding-characters question three fundamental criteria used to define the concept of character: the presence of a media signifier, the integration of the character in a fictional universe and the fact that characters are human-like. In order to describe precisely how they escape these definitional frameworks, we will first distinguish two main categories of guiding-characters: interfacial characters and tutorial avatars. Secondly, we will discuss the narrative specificities of these entities: by mediating between the game universe and the player's empirical gesture, they are indeed intrinsically metaleptic and they therefore do not exist on the same plane as the other characters. On the basis of this observation, we will differentiate tutorial avatars according to the rhetorical strategies they employ to negotiate with their transgressive nature: whether they conceal it (via tutorial metaphors), whether they leave it apparent (via raw metalepses) or whether they underline it (via fictionalized metalepses).

Cet article propose d'étudier les tutoriels vidéoludiques depuis une perspective narratologique et rhétorique. Plus précisément, il s'intéresse aux incarnations narratives du tutoriel que sont les «personnages-guides », ces mascottes qui accompagnent la progression du joueur en lui dictant 
la marche à suivre à chaque étape du jeu. Ces êtres hybrides représentent en effet une porte d'entrée idéale pour étudier les mécanismes de narrativisation des règles qui sont à l'œuvre au sein du médium, puisqu'ils combinent une fonction utilitaire (révéler le mode d'emploi du jeu) et une fonction narratologique (faire progresser le récit). À travers l'analyse d'une série d'exemples, cette recherche visera à démontrer que les personnages-guides remettent en question trois critères fondamentaux utilisés pour définir le concept personnage : la présence d'un signifiant médiatique, l'intégration du personnage dans un univers fictionnel et le fait que les personnages soient conçus sur le modèle de l'humain. Pour décrire précisément en quoi ils échappent à ces cadres définitionnels, nous distinguerons d'abord deux grandes catégories de personnagesguides: les personnages interfaciels et les avatars tutoriels. Dans un second temps, nous aborderons les spécificités narratives de ces entités : en servant de médiation entre l'univers ludique et le geste empirique du joueur, celles-ci sont intrinsèquement métaleptiques et ne se situent donc pas tout à fait sur le même plan d'existence que les autres personnages. Sur la base de cette observation, nous différencierons les avatars tutoriels en fonction des stratégies rhétoriques qu'ils emploient pour négocier avec leur nature transgressive : qu'ils la dissimulent (via des métaphores tutorielles), qu'ils la laissent apparente (via des métalepses brutes) ou qu'ils la soulignent (via des métalepses fictionnalisées).

\section{INDEX}

Mots-clés: Personnage ; Tutoriel ; Sciences du jeu ; Métalepse ; Fiction

Keywords: Character ; Tutorial ; Game studies ; Metalepsis ; Fiction

\section{AUTHOR}

\section{FANNY BARNABÉ}

Fanny Barnabé est Chargée de recherches FNRS à l'Université de Liège et membre du Liège Game Lab. Ses recherches portent sur la narration vidéoludique (à laquelle elle a consacré l'ouvrage Narration et jeu vidéo: Pour une exploration des univers fictionnels, paru aux Presses Universitaires de Liège), sur les différentes formes de détournement du jeu vidéo (sujet de sa thèse de doctorat) et sur les tutoriels de jeu vidéo. En 2017-2018, elle a réalisé un séjour postdoctoral d'un an à Kyoto, au sein du Ritsumeikan Center for Game Studies. 